\title{
SOME CHARACTERIZATIONS OF SEMIPRIME GOLDIE RINGS
}

\author{
by S. R. LÓPEZ-PERMOUTH, S. TARIQ RIZVI and M. F. YOUSIF
}

\author{
(Received 12 May, 1992)
}

1. Introduction. The notation in this paper will be standard and it may be found in [3], for example. In particular, the notation $A \subset \subset^{\prime} B$ stands for the statement " $A$ is an essential submodule of $B$ ". As is customary, we say that a ring $R$ is a Goldie ring when $R$ is both left and right Goldie. Similarly, a ring is noetherian if and only if it is both right and left noetherian, etc.

Semiprime Goldie rings constitute a much studied and well-known family of rings. In this paper we provide characterizations of semiprime Goldie rings in terms of their right ideals and in terms of their nonsingular right modules (Theorem 3.9). In particular, a ring $R$ is semiprime Goldie if and only if every right ideal of $R$ is weakly-injective, if and only if $R$ is right nonsingular and every nonsingular right $R$-module is weakly-injective. The above statements, with weakly-injective replaced by injective, are characterizations of semisimple artinian rings. So, Theorem 3.9 points out one analogy between semiprime Goldie rings and semisimple artinian rings.

In Section 4 we apply our results to the study of weakly-semisimple rings. Among other new characterizations of right weakly-semisimple rings, we obtain that a ring $R$ is right weakly-semisimple if and only if every essential submodule of every cyclic right $R$-module is weakly-injective (Theorem 4.1 ), if and only if every finitely generated right $R$-module is compressible (Theorem 4.2), if and only if $R$ is semiprime Goldie and every singular right $R$-module is weakly-injective (Theorem 4.5). In studying the problem of symmetry (is every right weakly-semisimple ring also left weakly-semisimple?), we obtain that a right weakly-semisimple ring is left weakly-semisimple if and only if every finitely generated singular left module is compressible (Theorem 4.6). Also, we prove that a right weakly-semisimple and left hereditary ring is left weakly-semisimple (Theorem 4.7).

Right weakly-semisimple rings are right QI-rings. That is, if $R$ is a weakly-semisimple ring then every quasi-injective right $R$-module is injective (see [4], [5], [6], [9], [10], [14], etc. for background on right QI-rings). If $R$ is hereditary and noetherian then $R$ is a right weakly-semisimple ring if and only if it is a right QI-ring ([12]). The symmetry problem for QI-rings is open to this day even for rings all of whose proper cyclic right modules are injective (right PCI rings).

2. Preliminaries. Given an arbitrary ring $R$ and $R$-modules $M$ and $N$, we say that $M$ is weakly $N$-injective if and only if every map $\varphi: N \rightarrow E(M)$ from $N$ into the injective hull $E(M)$ of $M$ may be written as a composition $\sigma \circ \hat{\varphi}$, where $\hat{\varphi}: N \rightarrow M$ and $\sigma: M \rightarrow E(M)$ is a monomorphism. This is equivalent to saying that for every map $\varphi: N \rightarrow E(M)$ there exists a submodule $X$ of $E(M)$, isomorphic to $M$, such that $\varphi(N)$ is contained in $X$. In particular, $M$ is weakly $R$-injective if and only if for every $x \in E(M)$ there exists $X \subset E(M)$ such that $x \in X \cong M$. We say that $M$ is weakly-injective if and only if it is weakly $N$-injective for every finitely generated module $N$. Clearly, $M$ is weakly-injective if and only if for every finitely generated submodule $N$ of $E(M)$ there exists $X \subset E(M)$ such that $N \subset X \cong M$.

S. T. Rizvi wishes to acknowledge the partial support he received from NSF through grant No. INT-9003849. M. F. Yousif would like to acknowledge the partial support of this work under the Ohio State University-Lima campus Research grant No. 9240.' 
Any weakly $N$-injective module $M$ satisfies the closely related property that for every submodule $K$ of $N$, if $N / K$ embeds in $E(M)$ then $N / K$ embeds in $M$. Following [11], we refer to any such module as being $N$-tight. If $M$ is $N$-tight for every finitely generated module $N$, we say that $M$ is tight. Following [12], a ring $R$ satisfying that every right $R$-module is weakly-injective will be referred to as a right weakly-semisimple ring. It is not hard to see, following the arguments in [11] and [12], that a ring $R$ is weaklysemisimple if and only if every right $R$-module is tight.

Weakly-injective (tight) modules are closed under finite direct sums and under essential extensions. However, they remarkably fail to be closed under direct summands [11]. In fact, summands of weakly-injective (tight) modules are weakly-injective (tight) if and only if $R$ is a right weakly-semisimple ring [16]. For any right $R$-module $N$ over a right noetherian ring $R$, arbitrary direct sums of weakly $N$-injective right modules are weakly $N$-injective. Also, all direct sums of (weakly-) injective right modules over a ring $R$ are weakly-injective if and only if every cyclic right $R$-module has finite Goldie dimension [1]. Rings for which every cyclic right module has finite Goldie dimension are referred to as right q.f.d. rings. Every tight right module over a right q.f.d. ring is weakly-injective [16]. For more on q.f.d. rings, see [7], [15] and [17].

The following series of results from various sources is presented here in order to make it easier to refer to them later in the paper. We start with a statement that makes precise the connection between tightness and weak-injectivity.

Leмma 2.1. Given right modules $M$ and $N, M$ is weakly $N$-injective if and only if for every submodule $Q$ of $N$ and for every monomorphism $\sigma: N / Q \rightarrow E(M)$ :

(1) there exists a monomorphism $\sigma^{\prime}: N / Q \rightarrow M$, and

(2) for every complement $K$ of $\sigma^{\prime}(N / Q)$ in $M$ there exists $K^{\prime} \subset E(M)$ such that $K^{\prime} \cap \sigma(N / Q)=0$ and $K^{\prime} \cong K$.

In particular, a uniform module is weakly-injective if and only if it is tight.

Proof. This is Lemma 3.1 and Corollary 1.4 from [12].

The next lemma describes some instances in which weak-injectivity may be replaced by weak injectivity relative to $R^{2}$.

Lemma 2.2. Let $M$ be a right $R$-module. Then

(1) if $M$ is cyclic then $M$ is weakly-injective if and only if it is weakly $R^{2}$-injective, and

(2) if $M$ has finite Goldie dimension then every submodule of $M$ is weakly-injective if and only if every submodule of $M$ is weakly $R^{2}$-injective.

Proof. Part (1) is Lemma 1.12 in [12]. In order to prove (2), assume $M$ is a finite Goldie dimensional right module and that every submodule of $M$ is weakly $R^{2}$-injective. Let $N$ be a submodule of $M$. Since $N$ is itself finite dimensional there exists a finite direct sum of uniform cyclic submodules $\bigoplus_{i=1}^{n} U_{i}$ which is an essential submodule of $N$. Each $U_{i}$ is cyclic and weakly $R^{2}$-injective, and hence weakly-injective by (1). So, the sum $\bigoplus_{i=1}^{n} U_{i}$ is also weakly-injective. It follows then that $N$, being an essential extension of a weakly-injective module, is weakly-injective.

We continue with some illustrative examples which will be useful in the sequel. 
Examples 2.3. (1) Let $R$ be the ring of endomorphisms of an infinite dimensional vector space $V$ over a field $F$. Then $M=\operatorname{Soc}\left(R_{R}\right) \oplus R$ is a tight module which is not weakly-injective.

(2) Let $R$ be a domain. Then $R$ is weakly $R$-injective if and only if $R$ is right Ore. On the other hand, $R$ is weakly $R^{2}$-injective if and only if it is two sided Ore.

(3) Let $S$ be a full matrix ring over a domain $R$. Then $S$ is a right weakly-injective ring if and only if $S$ is right weakly $S$-injective, if and only if $R$ is a right weakly-injective ring.

Proof. As is well known, $R$ is self injective and $\operatorname{Soc}\left(R_{R}\right) \subset^{\prime} R_{R}$. Also, there exists a simple right $R$-module $S$ and an infinite cardinal $\aleph$ such that $\operatorname{Soc}\left(R_{R}\right) \cong S^{(\aleph)}$. So, $E\left(\operatorname{Soc}\left(R_{R}\right)\right)=R$ and

$$
E(M)=E\left(\operatorname{Soc}\left(R_{R}\right)\right) \oplus R \cong E\left(\operatorname{Soc}\left(R_{R}\right) \oplus \operatorname{Soc}\left(R_{R}\right)\right) \cong E\left(\operatorname{Soc}\left(R_{R}\right)\right)=R .
$$

Obviously, since $E(M)$ is isomorphic to a submodule of $M, M$ is tight. On the other hand, $M$ is not even weakly $R$-injective, as follows: let $\sigma: M \rightarrow E(M)$ be the embedding of $M$ into its injective hull. By the injectivity of $E(M)$, and since $M$ is an essential submodule of $R, \sigma$ may be extended to a monomorphism $\hat{\sigma}: R \rightarrow E(M)$. Consider the element $x=\hat{\sigma}(1) \in E(M)$. If there were an $X \subset E(M)$ such that $\hat{\sigma}(R) \subset X \cong M$, one would have that $X=A \oplus B$, where $A \cong \operatorname{Soc}\left(R_{R}\right)$ and $B \cong R$. This would imply (since $\hat{\sigma}(R) \subset^{\prime} E(M)$ ) that $A \subset \hat{\sigma}(R) \subset X$. However, since $A$ is a direct summand of $X$, this would yield that $A$ is a direct summand of the cyclic module $\hat{\sigma}(R)$. This is a contradiction in view of the fact that $A$ is an infinite direct sum. So, no such $X$ may exist and therefore, $M$ is not weakly-injective. This concludes the proof of (1). (For more on this, see Section 3 of [16].)

(2) and (3) are Example 1.15(i) of [12] and Theorem 3.4 of [2], respectively.

Following [13], a module is said to be compressible if it is embeddable in every one of its essential submodules.

Lemma 2.4. A semiprime right Goldie ring $R$ is left Goldie if and only if every finitely generated nonsingular right $R$-module is compressible.

Proof. See Theorem 2.2.15 in [13].

LеммA 2.5. A right finite dimensional right hereditary ring is right noetherian.

Proof. See [9, Lemma 2.24].

LEMMA 2.6. If the ring $R$ is two-sided noetherian and left hereditary then $R$ is also right hereditary.

Proof. See [8, Corollary 8.18].

3. Semiprime Goldie rings. In this section we will provide characterizations of semiprime Goldie rings in terms of their right ideals and their right modules. We show that a ring $R$ is semiprime Goldie if and only if every right (left) ideal is weakly-injective. In turn, this is equivalent to the requirements for $R$ to be right nonsingular and for every nonsingular right $R$-module to be weakly-injective.

LEMMA 3.1. An arbitrary ring $R$ is right nonsingular if either the right singular ideal $Z_{\mathrm{r}}(R)$ of $R$ or every essential right ideal of $R$ is $R$-tight. 
Proof. Suppose $Z_{\mathrm{r}}(R)$ is $R$-tight. Write $E\left(Z_{\mathrm{r}}(R)\right) \oplus K=E(R)$ and consider $1 \in R \subset$ $E(R)$ as a sum $1=a+b$, where $a \in E\left(Z_{\mathrm{r}}(R)\right)$ and $b \in K$. By the $R$-tightness of $Z_{\mathrm{r}}(R)$, $E\left(Z_{\mathrm{r}}(R)\right)$ is singular, indeed $E\left(Z_{\mathrm{r}}(R)\right)=Z(E(R))$. So, there exists an essential right ideal $I \subset^{\prime} R$ such that $a l=0$. But then $b I=I$ and hence $I \subset K$ is nonsingular. Since $0=Z(I)=I \cap Z_{\mathrm{r}}(R)$ and $I$ is essential in $R$, we conclude that $Z_{\mathrm{r}}(R)=0$, as claimed.

Assume now that every essential right ideal of $R$ is $R$-tight. There exists a right ideal $K$ of $R$ such that $Z_{\mathrm{r}}(R) \oplus K$ is essential in $R$ and thus $R$-tight. Consider $1 \in E(R)=$ $E\left(Z_{\mathrm{r}}(R) \oplus K\right)$. By the $R$-tightness of $Z_{\mathrm{r}}(R) \oplus K$ there exists an embedding $\varphi: R \rightarrow$ $Z_{\mathrm{r}}(R) \oplus K$. Write $\varphi(1)=a+b, a \in Z_{\mathrm{r}}(R), b \in K$. Since $Z_{\mathrm{r}}(R)$ is singular there exists $I \subset{ }^{\prime} R_{R}$ such that $a I=0$, but then $\varphi(I)=b I$ and hence $\varphi(I) \subset K$. So $\varphi(I)$ and thus $I$ is nonsingular. We can then conclude, as in the previous proof, that $Z_{\mathrm{r}}(R)=0$.

LEMMA 3.2. Let $M$ be a finitely generated right $R$-module generated by $n$ elements. If every essential submodule of $M$ is weakly $R^{n}$-injective then $M$ has finite Goldie dimension.

Proof. Suppose $M$ is not finite dimensional. Then there exists an essential submodule $A=\bigoplus_{i=1}^{\infty} y_{i} R \subset^{\prime} M$, for some nonzero $y_{i} \in M$. Since $A$ is weakly $R^{n}$-injective, there exists a submodule $X$ of $E(A)=E(M)$, such that $M \subset X \cong A$. We may then write $X=\bigoplus_{i=1}^{\infty} y_{i}^{\prime} R$, where $y_{i}^{\prime} R \cong y_{i} R$. Since $M$ is finitely generated, $M \subset \bigoplus_{i=1}^{k} y_{i}^{\prime} R$, for some $k \in \mathbb{Z}^{+}$. Thus $M \cap y_{i}^{\prime} R=0$ for all $i>k$, which contradicts the essentiality of $M$ in $E(M)$.

COROLLARY 3.3. If every essential right ideal of the ring $R$ is weakly $R$-injective then $R$ has finite right Goldie dimension.

Proof. Clear.

Lemma 3.4. If every essential right ideal of the ring $R$ is $R$-tight then $R$ is semiprime.

Proof. Let $I$ be a nonzero ideal of $R$, and let $I^{\prime}$ be a right complement of $I$. Then $I \oplus I^{\prime} \subset^{\prime} R$. As $l \oplus I^{\prime}$ is $R$-tight, there exists an embedding $\varphi: R \rightarrow I \oplus I^{\prime}$. Let $x=\varphi(1)=$ $a+b, a \in I, b \in I^{\prime}$. For any nonzero element $y \in I, \varphi(y)=\varphi(1) y=x y=(a+b) y=a y$, since $b y \in I \cap I^{\prime}=0$. Therefore, $a y \in I^{2}$ and $a y \neq 0$. So $R$ is semiprime as claimed.

Proposition 3.5. If every (essential) right ideal of the ring $R$ is weakly-injective then $R$ is semiprime Goldie.

Proof. From Lemma 3.1, Corollary 3.3 and Lemma 3.4, we obtain that $R$ is semiprime right Goldie. It follows therefore that the injective hull of $R$ as a right module is its complete ring of right quotients $Q$. Let $q \in Q$. Since $R$ is right weakly-injective there exists $q^{\prime} \in Q$ such that the right annihilator of $q^{\prime}$ is 0 and $1, q \in q^{\prime} R$. It follows that $q^{\prime}=r^{-1}$ for some $r \in R$ and there exists $s \in R$ such that $q=r^{-1} s$. Therefore, $Q$ is also a left ring of quotients for $R$ and hence $R$ is left Goldie.

Example 3.6. Note that in Proposition 3.5, we only use weak $R$-injectivity for all proper (essential) right ideals of $R$. However, the requirement for $R$ to be weaklyinjective is needed: let $R$ be any principal right ideal domain which is not left Ore. Then every right ideal of $R$ (being isomorphic to $R$ ) is weakly $R$-injective (Example 2.3(1)). However, $R$ is a semiprime right Goldie ring which is not left Goldie. 
The following proposition was proved in [12] for indecomposable injective modules.

Proposition 3.7. Let $E$ be an injective right $R$-module. Then, the following conditions are equivalent:

(1) every submodule of $E$ is tight;

(2) every finitely generated submodule of $E$ is compressible.

Proof. Let us assume that every submodule of $E$ is tight. Let $N$ be a finitely generated submodule of $E$ and let $A \subset^{\prime} N$. Since $A$ is tight and $E(A)=E(N), N$ embeds in $A$. Conversely, let $M$ be any submodule of $E$, and let $A$ be any finitely generated submodule of $E(M) \subset E$. Then $0 \neq A \cap M \subset^{\prime} A$ holds, and since $A$ is compressible, we obtain a monomorphism from $A$ into $A \cap M \subseteq M$. Thus every finitely generated submodule of $E(M)$ embeds in $M$, as claimed.

Proposition 3.8. Every nonsingular module over a semiprime Goldie ring is weakly injective.

Proof. Proposition 3.7 and Lemma 2.4 yield that every nonsingular module over a semiprime Goldie ring is tight. In particular, every uniform nonsingular module is weakly-injective. It follows then that every finite dimensional nonsingular module is weakly-injective. Let $M$ be an arbitrary nonsingular right $R$-module. Since $R$ is semiprime Goldie there exists $M^{\prime}=\bigoplus_{i \in I} U_{i}$, a direct sum of (possibly infinitely many) nonzero uniform submodules of $M$, which is essential in $M$. Let $N$ be a finitely generated submodule of $E(M)=E\left(M^{\prime}\right)$. Since $M^{\prime}$ is tight, there exists an embedding $\varphi: N \rightarrow M^{\prime}$. Now, $\varphi(N)$ is finitely generated. Thus there exists a finite subset $J \subset I$ such that $\varphi(N) \subset \bigoplus_{i \in J} U_{i}$. It follows that, for some $K, E(\varphi(N)) \oplus K=\bigoplus_{i \in J} E\left(U_{i}\right)$. By the Krull-Schmidt theorem, we then get that $E(\varphi(N)) \cong \bigoplus_{i \in I_{1}} E\left(U_{i}\right)$ for some $l_{1} \subset J$. So, there exists a new embedding $\varphi_{1}: N \rightarrow$ $E(M)$ such that $\varphi_{1}(N) \subset^{\prime} \bigoplus_{i \in I_{1}} E\left(U_{i}\right)$. Now, $\bigoplus_{i \in I_{i}} U_{i}$, being finite dimensional, is weaklyinjective. Thus, there exists $X_{1} \subset E\left(\bigoplus_{i \in I_{1}} U_{i}\right)$ such that $X_{1}=\bigoplus_{i \in I_{1}} V_{i}$ and $U_{i} \cong V_{i}$ for all $i \in I_{1}$, and $\varphi_{1}(N) \subset^{\prime} \bigoplus_{i \in I_{1}} V_{i}$. Note that $\varphi_{1}(N) \subset \bigoplus_{i \in I_{1}} V_{i} \oplus \underset{i \in I-I_{1}}{\bigoplus_{i}} U_{i} \cong \bigoplus_{i \in I} U_{i}$. Summarizing, we have that, for some $K_{1} \subset E(M)$,

$$
\begin{aligned}
E(N) \oplus K_{1} & =E(M)=E\left(\varphi_{1}(N)\right) \oplus E\left(\bigoplus_{i \in I-I_{1}} U_{i}\right) \\
& =E\left(\bigoplus_{i \in I_{1}} E_{i} \oplus \bigoplus_{i \in I-l_{1}} E_{i}\right),
\end{aligned}
$$

where $E_{i}$ equals $E\left(V_{i}\right)$ (if $\left.i \in I_{1}\right)$ or $E\left(U_{i}\right)$ (if $\left.i \in I-I_{1}\right)$. On the other hand, there exists a collection $\left\{T_{\lambda}\right\}_{\lambda \in \Lambda}$ of indecomposable injectives such that $K_{1}=E\left(K_{1}\right)=E\left(\bigoplus_{\lambda \in \Lambda} T_{\lambda}\right)$. Since $E(N) \cong E\left(\varphi_{1}(N)\right)$, we may write $E(N)=\bigoplus_{i \in I_{1}} W_{i}$, with $W_{i} \cong E_{i}$ for all $i \in I_{1}$, thus

$$
E(M)=E(N) \oplus K_{1}=E\left(\bigoplus_{i \in L_{1}} W_{i} \oplus \bigoplus_{\lambda \in \Lambda} T_{\lambda}\right)
$$


It follows then, by Corollary 4.2 in [19], that

$$
\bigoplus_{i \in I_{1}} W_{i} \oplus \underset{\lambda \in \Lambda}{\bigoplus} T_{\lambda} \cong \bigoplus_{i \in I_{1}} E_{i} \oplus \bigoplus_{i \in I-I_{i}} E_{i}
$$

Then, using the Azumaya-Krull-Schmidt theorem we get a bijection $\sigma: I-I_{1} \rightarrow \Lambda$ such that, for every $i \in I-I_{1}, E_{i} \cong V_{\sigma(i)}$. Without loss of generality let us assume that $\Lambda=I-I_{1}$ and that $\sigma$ is the identity map. Let $\Psi_{i}: E_{i} \rightarrow T_{i}$ be an isomorphism then $\Psi_{i}\left(U_{i}\right) \subset^{\prime} T_{i}$. Consider the obvious isomorphism $\Phi$ between $\varphi_{1}(N) \oplus \underset{i \in I-I_{1}}{\bigoplus} U_{i}$ and $N \oplus \underset{i \in I-I_{1}}{\bigoplus} \Psi_{i}\left(U_{i}\right)$ as a map

$$
\Phi: \varphi_{1}(N) \oplus \underset{i \in I-l_{1}}{\bigoplus} U_{i} \rightarrow E(M) .
$$

Since the domain of $\Phi$ embeds as an essential submodule of $M$, the injectivity of $E(M)$ yields the existence of a monomorphism $\hat{\Phi}: M \rightarrow E(M)$ extending $\Phi$ for which $N \subset \hat{\Phi}(M)$. Thus $M$ is weakly-injective, as claimed.

We are now ready to prove the main theorem of this section.

THEOREM 3.9. The following conditions are equivalent over an arbitrary ring $R$ :

(1) $R$ is semiprime Goldie;

(2) $R$ is right nonsingular and every nonsingular right $R$-module is weakly-injective;

(3) every right ideal of $R$ is weakly-injective;

(4) every essential right ideal of $R$ is weakly-injective; and

(5) every essential right ideal of $R$ is weakly $R$-injective and $R_{R}$ is weakly-injective.

Proof. Semiprime Goldie rings are (right and left) nonsingular. Therefore Proposition 3.8 yields that (1) implies (2). Clearly, (2) implies (3), (3) implies (4) and (4) implies (5). The implication (5) $\Rightarrow(1)$ is Proposition 3.5 (see also Example 3.6).

Since condition (1) in Theorem 3.9 is left-right symmetric, the remaining conditions (2)-(5) may be replaced by their left-handed versions.

EXAMPLE 3.10. In view of condition (5) in Theorem 3.9 and of the proof of Proposition 3.5, one may be tempted to conjecture that a ring $R$ is semiprime right Goldie if and only if every right ideal of $R$ is weakly $R$-injective. While the latter condition is indeed sufficient in order for $R$ to be semiprime and right Goldie, the converse is not true: let $S$ be a full matrix ring over a principal right ideal domain $R$ which is not left Ore, then $S$ is prime and right noetherian but $S$ is not weakly $S$-injective (Example 2.3(3)).

One can actually characterize semiprime Goldie rings in a manner analogous to Theorem 3.9 but in terms of weak $R^{2}$-injectivity.

Proposition 3.11. The following conditions for a ring $R$ are equivalent:

(1) $R$ is semiprime Goldie;

(2) $R$ is right nonsingular and every nonsingular right $R$-module is weakly $R^{2}$-injective and

(3) every (essential) right ideal of $R$ is weakly $R^{2}$-injective.

Proof. Theorem 3.9 shows that (1) implies (2). The implication (2) implies (3) is obvious. The implication (3) $\Rightarrow(1)$ follows from condition (5) in Theorem 3.9.

It is tempting to provide similar characterizations for semiprime Goldie rings in terms of tightness. This is the subject of our next proposition. 
Proposition 3.12. For a ring $R$ with finite right Goldie dimension the following statements are equivalent:

(1) $R$ is semiprime Goldie;

(2) $R$ is right nonsingular and every nonsingular right module is tight;

(3) $R$ is right nonsingular and every finitely generated nonsingular right $R$-module is compressible; and

(4) every right ideal of $R$ is tight.

Proof. (1) implies (2) in light of Theorem 3.9 and since weakly injective modules are tight. Proposition 3.7 yields that (2) and (3) are equivalent. Clearly, (2) implies (4). Since we are assuming that $R$ has finite right Goldie dimension, every right ideal of $R$ contains as an essential submodule a finite direct sum of uniform right ideals. Assuming (4), each one of these uniform right ideals is tight, hence weakly-injective (Lemma 2.1). It follows then that every right ideal is weakly-injective and therefore $R$ is semiprime Goldie, by Theorem 3.9.

The equivalence of conditions (1) and (3) above generalizes Lemma 2.4 since we obtained it with a weaker hypothesis.

4. Weakly-semisimple rings. In this section we apply our results to provide several new characterizations of right weakly-semisimple rings and to explore the symmetry problem for weakly-semisimple rings.

Among other characterizations, Theorem 2.5 in [12] shows that a ring $R$ is right weakly-semisimple if and only if it is right noetherian and every cyclic right $R$-module is weakly-injective (condition (iii)). It is open whether or not one may remove the noetherian condition from the above criterion, the next theorem is one step in that direction.

THEOREM 4.1. A ring $R$ is right weakly-semisimple if and only if every essential submodule of every cyclic right $R$-module is weakly $R^{2}$-injective.

Proof. Necessity is obvious. Conversely, let every essential submodule of every cyclic right $R$-module be weakly $R^{2}$-injective. It follows that every simple right module is injective, i.e. $R$ is a right $\mathrm{V}$-ring. Next, in view of Lemma 3.2, for any right ideal $I$ of $R$, the quotient module $R / I$ is finite dimensional. So, $R$ is a right q.f.d. ring as well as a right $V$-ring. It follows that every completely reducible right module is weakly $R^{2}$-injective and hence injective. This implies that $R$ is right noetherian. We can now apply Theorem 2.5 (condition (iii)) in [12] to get that $R$ is right weakly-semisimple.

Theorem 2.5 in [12] also characterizes right weakly-semisimple rings as being those right noetherian rings for which every finitely generated uniform right $R$-module is compressible (condition (v)). Our next theorem relates strongly to that result. We remove the explicit condition of right noetherianness and, in exchange, require all finitely generated right modules to be compressible.

THEOREM 4.2. A ring $R$ is right weakly-semisimple if and only if every finitely generated right $R$-module is compressible.

Proof. If every finitely generated right $R$-module is compressible then it follows from Proposition 3.7 that every right $R$-module is tight. 
Remark 4.3. $A$ ring $R$ is weakly-semisimple if and only if every singular right $R$-module and every nonsingular right $R$-module are weakly-injective (tight).

Proof. Necessity is obvious. Conversely, let $M$ be any right $R$ module. Let $Z(M)$ be the singular submodule of $M$, and let $K$ be a right complement of $Z(M)$ in $M$. Then $Z(M) \oplus K \subset^{\prime} M$ and $Z(M) \oplus K$ is weakly-injective (tight). Therefore, $M$ is weaklyinjective (tight), hence $R$ is right weakly-semisimple.

Before our next characterization we need the following lemma.

LEMMA 4.4. The following conditions on a ring $R$ are equivalent:

(1) $R$ is right nonsingular and every finitely generated singular right $R$-module is compressible;

(2) every singular right $R$-module is tight.

Proof. Over a right nonsingular ring, right singular modules are closed under essential extensions. The proof of the implication $(1) \Rightarrow(2)$ follows then directly from Proposition 3.7. Conversely, if every singular right $R$-module is tight then, in particular, completely reducible right modules are tight hence injective. Using Corollary 2.14 from [17], we get that $R$ is right nonsingular. The rest of the proof follows, once again, from Proposition 3.7.

THEOREM 4.5. The following are equivalent for an arbitrary ring $R$ :

(1) $R$ is right weakly-semisimple;

(2) $R$ is right $Q I$ and left Goldie and each finitely generated singular right module is compressible;

(3) $R$ is right $Q I$ and left Goldie and each singular right module is tight; and

(4) $R$ is semiprime Goldie and each singular right module is tight.

Proof. $(1) \Leftrightarrow(2)$. Since every weakly-injective quasi-injective right module is injective (Lemma 1.9 in [12]), $R$ is a right QI ring. That $R$ is left Goldie and every finitely generated singular right module is compressible follows from Theorem 3.9 and Lemma 4.4 .

The implication $(2) \Rightarrow(3)$ is clear from Lemma 4.4 .

Also, $(3) \Rightarrow(4)$ is obvious since every right QI ring is a right noetherian right V-ring. Finally, the implication (4) $\Rightarrow(1)$ follows from Proposition 3.8 and Remark 4.3.

Let us turn next to the symmetry problem.

THEOREM 4.6. A right weakly-semisimple ring $R$ is left weakly-semisimple if and only if every finitely generated singular left $R$-module is compressible.

Proof. A right weakly-semisimple ring $R$ is nonsingular. If $R$ is also left weaklysemisimple then, in particular, all singular left $R$-modules are tight. In light of Lemma 4.4 , this is equivalent to the requirement for every finitely generated singular left $R$-module to be compressible. Conversely, since $R$ is right weakly-semisimple, $R$ is semiprime Goldie. Now, the assumption that every finitely generated singular left module is compressible is equivalent to the requirement for every singular left $R$-module to be tight (Lemma 4.4). So, in light of Theorem 4.5 (condition (4)), we conclude that $R$ is left weakly-semisimple. 
The property of being QI is known to be symmetric [6] and equivalent to weak-semisimplicity [12] if the ring $R$ is assumed to be hereditary and noetherian (on both sides). The following result generalizes Theorem 3.1 in [12] by obtaining symmetry for weak-semisimplicity out of one-sided heredity.

THEOREM 4.7. A right weakly-semisimple and left hereditary ring is left weaklysemisimple.

Proof. If $R$ is right weakly-semisimple, $R$ is left finite dimensional and right noetherian. Then, by Lemma $2.5, R$ is also left noetherian. Lemma 2.6 now yields that $R$ is also right hereditary. Since $R$ is hereditary and noetherian on both sides, our result follows from Theorem 3.1 in [12].

\section{REFERENCES}

1. A. H. Al-Huzali, S. K. Jain and S. R. López-Permouth, Rings whose cyclics have finite Goldie dimension, to appear in J. Algebra.

2. A. H. Al-Huzali, S. K. Jain and S. R. López-Permouth, Weakly-injective rings and modules, Osaka J. Math. 29 (1992), 75-87.

3. F. W. Anderson and K. R. Fuller, Rings and categories of modules (Springer, 1974).

4. A. K. Boyle, Hereditary QI-rings, Trans. Amer. Math. Soc. 192 (1974), 115-120.

5. A. K. Boyle, Injectives containing no proper quasi-injective submodules, Comm. Algebra 4 (1976), 775-785.

6. A. K. Boyle and K. R. Goodearl, Rings over which certain modules are injective, Pacific J. Math. 58 (1975), 43-53.

7. V. P. Camillo, Modules whose quotients have finite Goldie dimension, Pacific J. Math. 69 (1977), 337-338.

8. A. W. Chatters and C. R. Hajarnavis, Rings with chain conditions (Pitman, 1980).

9. J. Cozzens and C. Faith, Simple Noetherian rings (Cambridge University Press, 1975). 113-119.

10. C. Faith, On hereditary rings and Boyle's conjecture, Arch. Math. (Basel) 27 (1976),

11. J. S. Golan and S. R. López-Permouth, QI-filters and tight modules, Comm. Algebra 19 (1991), 2217-2229.

12. S. K. Jain, S. R. López-Permouth and S. Singh, On a class of QI-rings, Glasgow Math. J. 34 (1992), 75-81.

13. A. V. Jategaonkar, Localization in Noetherian rings (Cambridge University Press, 1986).

14. K. A. Kosler, On hereditary and Noetherian $V$-rings, Pacific J. Math. 103 (1982), 467-473.

15. R. P. Kurshan, Rings whose cyclic modules have finitely generated socle, J. Algebra 15 (1970), 376-386.

16. S. R. López-Permouth, Rings characterized by their weakly-injective modules, Glasgow Math J. 34 (1992), 349-353.

17. S. S. Page and M. F. Yousif, Relative injectivity and chain conditions, Comm. Algebra 17 (1989), 899-924.

18. R. C. Schock, Dual generalizations of the Artinian and Noetherian conditions, Pacific J. Math. 54 (1974), 227-235.

19. R. B. Warfield, Decompositions of injective modules, Pacific J. Math. 31 (1969), 263-276.

S. R. López-PERMOUTH
OHIO UNIVERSITY
ATHENS
OHIO 4570

S. R. López-Permouth

OHIO UNIVERSITY

OHIO 4570
S. T. RIzVI AND M. F. YousiF

Ohio State University

LiMA

Оноо 45804 